\title{
Aplicação de gesso agrícola e especiação iônica da solução de um Latossolo sob sistema plantio direto
}

\author{
Gypsum application and ionic speciation of the solution from an Oxisol under no-till system
}

\author{
Fernando César Bachiega Zambrosi ${ }^{\mathrm{I}}$ Luís Reynaldo Ferracciú Alleoni ${ }^{\mathrm{II}}$ \\ Eduardo Fávero Caires ${ }^{\text {III }}$
}

\section{RESUMO}

O baixo teor de Ca e a toxicidade por Al são importantes limitações à produtividade das culturas em solos ácidos brasileiros. É possível aumentar o teor de Ca e reduzir a toxicidade por Al por meio da aplicação de gesso agrícola. No entanto, é importante melhor compreensão dos efeitos do gesso na especiação iônica da solução do solo. Avaliou-se a especiação iônica da solução do solo (extrato aquoso 1:1) de amostras de um Latossolo Vermelho distrófico textura argilosa, sob sistema plantio direto, cinco anos após a aplicação superficial de 3, 6 e $9 \mathrm{tha}^{-1}$ de gesso agrícola. A especiação foi realizada com auxílio do programa computacional Visual Minteq em amostras coletadas nas profundidades de 0-0,05; 0,05-0,1; 0,1-0,2 e 0,2-0,4m. Para o Al, as principais espécies químicas foram os complexos com carbono orgânico dissolvido (Al-COD) e com F (Al-F), enquanto o par iônico $\mathrm{AlSO}_{4}^{+}$ocorreu em pequena proporção. $O$ COD foi o principal ligante para $\mathrm{Mg}$ e Ca, mas em muito maior magnitude para este último, refletindo importante participação dos ânions orgânicos na dinâmica desses nutrientes. Em relação ao sulfato, apesar da formação dos pares iônicos com $\mathrm{Ca}, \mathrm{Mg}$ e $\mathrm{Al}\left(\mathrm{CaSO}_{4}{ }^{0}, \mathrm{MgSO}_{4}{ }^{0}\right.$ e $\left.\mathrm{AlSO}_{4}^{+}\right)$, houve a predominância da forma livre $\mathrm{S}-\mathrm{SO}_{4}{ }^{-2}$, enquanto o fosfato apresentou forte interação com o $\mathrm{Al} \mathrm{em}$ solução, formando a espécie química Al- $\mathrm{H}_{x} \mathrm{PO}_{4}{ }^{x}$.

Palavras-chave: par iônico, complexação, carbono orgânico dissolvido, alumínio, cátions, ânions.

\section{ABSTRACT}

Low Ca content and Al toxicity are important limitations for yield crops in Brazilian acids soils. Gypsum application may increase Ca contents and decrease Al toxicity. However, a better knowledge of the effects of gypsum on the

\begin{abstract}
ionic speciation of soil solution is very important. The ionic speciation of the soil solution (water extract 1:1) was evaluated in samples from a dystrophic clayey Rhodic Hapludox under no-till system, 5 years after surface application of gypsum (3; 6 and $9 t \mathrm{ha}^{-1}$ ). The ionic speciation was performed by using the Visual Minteq program in samples collected at the following depths: 0-0.05; 0.05-0.1; 0.1-0.2 and 0.2-0.4m. The main Al complexing agents in solution were with dissolved organic carbon (Al-DOC) and F (Al-F), while the ionic par AlSO + occurred in small proportion. Ca and $\mathrm{Mg}$ were complexed by DOC, and the effect was more pronounced for Ca, reinforcing the important participation the organic anions in the dynamic of these nutrients. Although sulphate had formed ionic pairs with $\mathrm{Ca}, \mathrm{Mg}$ and $\mathrm{Al}\left(\mathrm{CaSO}_{4}{ }^{\circ}, \mathrm{MgSO}^{\circ}\right.$ and $\left.\mathrm{AlSO}_{4}^{+}\right)$, the free form $\left(\mathrm{S}_{-} \mathrm{SO}_{4}{ }^{-2}\right)$ predominated; whille phosphate strongly interacted with Al in solution, forming the chemical specie Al$\mathrm{H}_{\mathrm{X}} \mathrm{PO}_{4}{ }^{\mathrm{x}}$.
\end{abstract}

Key words: ionic pair, complexation, dissolved organic carbon, aluminum, anions, cations.

\section{INTRODUÇÃO}

A deficiência de Ca e a toxicidade por Al são as principais barreiras químicas para o crescimento radicular, cujas conseqüências se manifestam pelo estresse nutricional e hídrico das plantas (RITCHEY et al., 1980). A correção do subsolo ácido pode ser feita por meio da calagem profunda. Contudo, essa prática necessita de revolvimento do solo, razão pela qual não é de interesse em áreas já estabelecidas com sistema

\footnotetext{
ICentro de Solos e Recursos Ambientais, Instituo Agronômico (IAC), Av. Barão de Itapura, 1481, 13001-970, Campinas, SP, Brasil. E-mail: zambrosi@iac.sp.gov.br.

"Departamento de Solos e Nutrição de Plantas, Escola Superior de Agricultura “Luiz de Queiroz” (ESALQ), Universidade de São Paulo (USP), Av. Pádua Dias, 11, 13418-900, Piracicaba, SP, Brasil.

IIIDepartamento de Ciência do Solo e Engenharia Agrícola, Universidade Estadual de Ponta Grossa (UEPG), Av. Carlos Cavalcanti, 4748, 84030-900, Ponta Grossa, PR, Brasil.
} 
plantio direto (SPD) (CAIRES et al., 1998). Uma alternativa para reduzir a toxicidade do $\mathrm{Al}$ e aumentar o teor de Ca em profundidade é a aplicação de gesso agrícola ( $\left.\mathrm{CaSO}_{4} \cdot 2 \mathrm{H}_{2} \mathrm{O}\right)$ (RAIJ et al., 1998).

Os principais objetivos dos trabalhos realizados com gesso em condições de solos tropicais têm sido observar as respostas de produtividade das culturas e a lixiviação de cátions ao longo do perfil. Porém, algumas vezes, apenas o estudo das formas trocáveis dos elementos não é suficiente para explicar as alterações no solo e o comportamento das plantas cultivadas. Desta maneira, tem havido maior interesse em estudos de especiação iônica da solução do solo, ou seja, a determinação da concentração das diferentes formas químicas de um elemento numa matriz, que juntas constituem a concentração total deste elemento na amostra (BARRA et al., 2000). A especiação é importante porque ajuda a compreender a variação da disponibilidade e da mobilidade dos elementos na solução do solo. Por exemplo, a biodisponibilidade de fósforo em ambientes aquáticos ou terrestres não é relacionada somente com sua concentração total na solução, mas também com a distribuição de suas espécies, como ortofosfato livre $\left(\mathrm{H}_{2} \mathrm{PO}_{4}^{-}, \mathrm{HPO}_{4}^{-2}, \mathrm{PO}_{4}^{-3}\right)$, formas orgânicas ou complexos coloidais (HENS \& MERCKX, 2002).

A possibilidade de o gesso alterar a constituição da solução do solo é, sobretudo, conseqüência dos incrementos dos teores de Ca e de $\mathrm{S}_{-} \mathrm{SO}_{4}^{-2}$, por serem os elementos presentes em maiores proporções nesse produto (32,6\% de $\mathrm{CaO}$ e $18,7 \%$ de S) (RAIJ, 1992). O sulfato e o cálcio se ligam a outros elementos, formando várias espécies químicas na solução do solo. A formação de complexos de um íon com outros de valência oposta (pares de íons) altera a função biológica do elemento químico, o que confirma a importância da especiação química para interpretação da disponibilidade de íons para as raízes das plantas (CHAVES et al., 1991). Esses autores, num experimento para avaliar absorção de $\mathrm{Ca}$ e $\mathrm{Al}$ por raízes de cafeeiro, após aplicação de $\mathrm{CaCO}_{3}, \mathrm{CaCl}_{2}, \mathrm{Ca}\left(\mathrm{NO}_{3}\right)_{2}, \mathrm{CaSO}_{4} \mathrm{e}$ $\mathrm{MgCO}_{3}$ ao solo, concluíram que a absorção de Ca pelas raízes das plantas diminuiu com a redução da valência do íon em solução, após a formação de pares de íons com nitrato $\left(\mathrm{CaNO}_{3}^{+}\right)$, cloreto $\left(\mathrm{CaCl}^{+}\right)$ou sulfato $\left(\mathrm{CaSO}_{4}^{0}\right)$.

Devido à importância de compreender a variação das espécies químicas dos íons em solução frente às práticas de manejo do solo, avaliou-se a distribuição das espécies de alumínio e de alguns nutrientes na solução de um Latossolo cultivado no SPD, cinco anos após a aplicação de gesso agrícola.

\section{MATERIAL E MÉTODOS}

Foram utilizadas amostras de um experimento de campo instalado em 1998, numa pastagem nativa sobre um Latossolo Vermelho distrófico textura argilosa, no município de Ponta Grossa/PR. Os resultados das análises químicas do solo, antes da instalação do experimento, realizadas segundo métodos descritos em PAVAN et al. (1992), indicavam para as camadas de 0-0,2 e 0,2-0,4m, respectivamente: $\mathrm{pH} \mathrm{CaCl}_{2} 4,6$ e 4,2; M.O. 31 e 21 $\mathrm{g} \mathrm{dm}^{-3}$; P (Mehlich 1) 0,3 e $0,1 \mathrm{mg} \mathrm{dm}^{-3} ; \mathrm{K}^{+} 3,6$ e 2,2; $\mathrm{Ca}^{+2} 25$ e $7 ; \mathrm{Mg}^{+2} 20$ e $8 ; \mathrm{Al}^{+3}$ 3 e $8 ; \mathrm{H}+\mathrm{Al} 77,6$ e $97,0 \mathrm{mmol}_{\mathrm{c}} \mathrm{dm}^{-3}$; saturação por bases (V\%) 38 e 15; saturação por alumínio (m\%) 6 e $32 \%$. As análises granulométricas (segundo métodos da EMBRAPA, 1997), para essas mesmas profundidades, revelaram: areia 290 e 300; silte 130 e 100; argila 580 e $600 \mathrm{~g} \mathrm{~kg}^{-1}$, respectivamente.

O delineamento experimental foi em blocos ao acaso, com três repetições, utilizando-se parcelas experimentais com área total de $56 \mathrm{~m}^{2}$. Foram aplicadas manualmente 3, 6 e 9t ha ${ }^{-1}$ de gesso agrícola em superfície, em outubro de 1998. A análise química desse produto revelou $17,9 \%$ de $\mathrm{S}_{-} \mathrm{SO}_{4}^{-2}, 23,4 \%$ de Ca e $0,5 \%$ de $\mathrm{P}_{2} \mathrm{O}_{5}$ total. No sistema de rotação de culturas, foram utilizadas soja (1998-1999), cevada (inverno de 1999), soja (1999-2000), trigo (inverno de 2000), soja (20002001), milho (2001-2002) e soja (2002-2003). A adubação utilizada na semeadura da soja foi de $75 \mathrm{~kg} \mathrm{ha}^{-1}$ de $\mathrm{P}_{2} \mathrm{O}_{5}$ e $\mathrm{K}_{2} \mathrm{O}$, no primeiro cultivo, e de $60 \mathrm{~kg} \mathrm{ha}^{-1}$ de $\mathrm{P}_{2} \mathrm{O}_{5} \mathrm{e}$ $\mathrm{K}_{2} \mathrm{O}$, nos outros três cultivos. Em cada cultivo de soja, também foram aplicados $15 \mathrm{~kg} \mathrm{ha}^{-1}$ de $\mathrm{S}$ na forma de superfosfato simples. A cevada recebeu $75 \mathrm{~kg} \mathrm{ha}^{-1} \mathrm{de} \mathrm{N}$ e $92 \mathrm{~kg} \mathrm{ha}^{-1}$ de $\mathrm{P}_{2} \mathrm{O}_{5}$, enquanto o trigo recebeu $130 \mathrm{~kg} \mathrm{ha}^{-1}$ de $\mathrm{N}, 66 \mathrm{~kg} \mathrm{ha}^{-1}$ de $\mathrm{P}_{2} \mathrm{O}_{5}$ e de $\mathrm{K}_{2} \mathrm{O}$. A adubação utilizada para o milho foi de $120 \mathrm{~kg} \mathrm{ha}^{-1}$ de $\mathrm{N}, 78 \mathrm{~kg} \mathrm{ha}^{-1} \mathrm{P}_{2} \mathrm{O}_{5}$ e de $90 \mathrm{~kg} \mathrm{ha}^{-1} \mathrm{~K}_{2} \mathrm{O}$. Na adubação de semeadura das culturas, foram utilizadas formulações comerciais contendo fontes solúveis em água, e a uréia em cobertura no cultivo das gramíneas.

As amostras de solo foram coletadas logo após a colheita da soja, em maio de 2003. Para compor uma amostra composta, coletaram-se com trado calador (2cm de diâmetro) 12 subamostras por parcela nas camadas de $0-0,05,0,05-0,1$ e $0,1-0,2 \mathrm{~m}$, e cinco subamostras com trado holandês ( $5 \mathrm{~cm}$ de diâmetro) para a camada de 0,2-0,4m de profundidade. Após a coleta, as amostras foram imediatamente secadas ao ar, destorroadas e passadas em peneira com malha de $2 \mathrm{~mm}$ de diâmetro e acondicionadas em recipientes plásticos. Foram armazenadas à sombra e à temperatura ambiente até a realização das análises. 
Para obtenção da solução do solo, empregou-se o método do extrato aquoso (WOLT, 1994), utilizando uma relação solo:água de 1:1. Foram tomados $20 \mathrm{~g}$ de solo em tubo de centrífuga de $50 \mathrm{~mL}$, aos quais foram adicionados $20 \mathrm{~g}$ de água ultrapura. Posteriormente, os tubos foram colocados em mesa agitadora horizontal, agitando-se por 15 minutos a 150rpm, permanecendo depois em descanso por $1 \mathrm{~h}$. A seguir, a solução foi agitada novamente por mais 5 minutos e centrifugada durante 30 minutos a 1.500rpm. Foram realizadas três extrações distintas para obtenção dos extratos de cátions, de ânions e de carbono orgânico dissolvido (COD). Na obtenção do extrato para leitura de cátions, a solução foi passada em membrana de celulose com $0,45 \mu \mathrm{m}$ de malha. Outro extrato foi passado em membrana de celulose com $0,22 \mu \mathrm{m}$ de malha para posterior determinação dos ânions. O extrato para leitura de COD foi obtido após a passagem do extrato em filtro de microfibra de vidro $\mathrm{GF} / \mathrm{F}$ com $0,7 \mu \mathrm{m}$ de malha, previamente calcinado a $500^{\circ} \mathrm{C}$ por seis horas e depois acondicionado em frascos também calcinados.

As filtragens para obtenção dos extratos foram realizadas com auxílio de suporte para filtros de 13mm de diâmetro para a fração cátions e ânions e de $25 \mathrm{~mm}$ para o COD. Esses suportes eram conectados em seringas plásticas de $60 \mathrm{~mL}$, nas quais eram colocados os extratos obtidos após centrifugação e filtrados mediante pressão manual. Imediatamente após a centrifugação, foi determinado o pH nas soluções. Os teores totais de $\mathrm{Ca}, \mathrm{Mg}, \mathrm{K}, \mathrm{Na}, \mathrm{Al}$, Fe e Mn foram determinados no ICP-OES, enquanto os de $\mathrm{N}_{-} \mathrm{NO}_{3}^{-}$, S$\mathrm{SO}_{4}^{-2}, \mathrm{P}_{-} \mathrm{H}_{\mathrm{x}} \mathrm{PO}_{4}^{\mathrm{x}}$, Cle F em cromatógrafo, e o COD no analisador de carbono Shimadzu 5000 A. Cobre e zinco não foram determinados porque seus teores estiveram abaixo do limite de detecção do ICP-OES.

A força iônica (I) da solução do solo foi calculada segundo SPOSITO (1989):

$$
I=1 / 2 \sum_{i=1}^{n} C i .(Z i)^{2}
$$

em que: Ci é a concentração $\left(\mathrm{mol} \mathrm{L}^{-1}\right)$ de cada íon da solução e $\mathrm{Zi}$ a sua valência.

A especiação química da solução do solo foi realizada com o auxílio do programa computacional Visual Minteq (GUSTAFSSON, 2004), a partir das concentrações totais $\left(\mathrm{mg} \mathrm{L}^{-1}\right)$ dos cátions e dos ânions inorgânicos, de COD, da I calculada (mol L $\left.{ }^{-1}\right)$ e do $\mathrm{pH}$ da solução, obtendo-se a distribuição percentual das espécies de cada elemento na solução do solo para cada dose de gesso e profundidade de amostragem.

\section{RESULTADOS E DISCUSSÃO}

Alguns resultados da solução do solo utilizados para realizar a especiação de alumínio, cálcio, magnésio, fósforo e enxofre na solução do solo sob SPD, até a profundidade de $0,4 \mathrm{~m}$, estão apresentados na tabela 1 . A solução foi caracterizada principalmente pela alta concentração de COD e pelos baixos valores de $\mathrm{pH}$, que apresentam efeitos marcantes na distribuição das espécies dos elementos em solução (CAMARGO et al., 2001).

A especiação do Al foi dividida entre: (i) forma livre $\left(\mathrm{Al}^{+3}\right)$; (ii) formas hidroxiladas $(\mathrm{Al}-\mathrm{OH}=$ $\left.\mathrm{Al}(\mathrm{OH})^{+2}+\mathrm{Al}(\mathrm{OH})^{+}+\mathrm{Al}(\mathrm{OH})_{3}^{0}\right)$; (iii) ligada ao sulfato $\left(\mathrm{AlSO}_{4}^{+}\right)$; (iv) complexada com o fluoreto $\left(\mathrm{Al}-\mathrm{F}=\mathrm{Al}(\mathrm{F})^{+2}\right.$ $\left.+\mathrm{Al}(\mathrm{F})^{+}+\mathrm{Al}(\mathrm{F})^{0}{ }_{3}\right)$, e, por fim, aquela complexada com o COD (Al-COD) (Tabela 2). Esta última foi a de maior ocorrência até a profundidade de $0,1 \mathrm{~m}$, para todas as doses de gesso, sendo que, a partir da camada subjacente, o complexo Al-F assumiu tal posição, ou apresentou valores percentuais semelhantes aos de Al-COD. A participação do COD na complexação do Al confirma a importância do acúmulo de resíduos orgânicos na minimização dos efeitos fitotóxicos desse elemento, sendo esta condição encontrada mais freqüentemente em solos sob SPD. A importância do gesso em controlar os efeitos fitotóxicos do $\mathrm{Al}$ em solução parece menor em sistemas de cultivos que favorecem o acúmulo e a manutenção de MO no solo. SALET et al. (1999) também observaram que a maior parte do Al solúvel no SPD apresentou-se complexada como Al-ligantes orgânicos, enquanto apenas a metade estava nessa forma no sistema convencional.

A ligação do fosfato com o Al foi de pequena magnitude e praticamente não ocorreu, devido à baixíssima concentração deste ânion na solução do solo (Tabela 3), corroborando os resultados de SALET et al (1999), em que a espécie de $\mathrm{Al}-\mathrm{H}_{\mathrm{x}} \mathrm{PO}_{4}{ }^{\mathrm{x}}$ foi menor que $0,1 \%$ do total das espécies desse cátion. As formas hidroxiladas, em conseqüência dos baixos valores de pH (Tabela 1), também ocuparam baixa proporção das espécies de Al. O incremento de sulfato na solução do solo via gesso (Tabela 3) não resultou no aumento da concentração do par iônico $\mathrm{AlSO}_{4}^{+}$, confirmado pela ausência de correlação significativa $(\mathrm{P}>0,05)$ entre os teores totais de $\mathrm{S}-\mathrm{SO}_{4}^{-2}$ e de $\mathrm{AlSO}_{4}^{+}$em todas as profundidades estudadas.

Quando o gesso é aplicado em condições de alta acidez, atribui-se ao par iônico $\mathrm{AlSO}_{4}^{+}$uma importante participação na amenização da toxicidade por Al. No entanto, esse papel do $\mathrm{S}_{-} \mathrm{SO}_{4}^{-2}$ não foi observado em grande intensidade, sendo o outro ânion inorgânico, o F-' muito mais importante. A formação do par iônico ou de complexo é determinada pela constante de ligação entre os elementos envolvidos. Dessa maneira, como o valor da constante entre o $\mathrm{Al}$ e o S-SO ${ }_{4}^{-2}$ é menor do que aquela formada entre $\mathrm{Al}$ e $\mathrm{F}$, a espécie Al- 
Tabela 1 - Valores de $\mathrm{pH}$, da força iônica (I) (mmol L $\left.{ }^{-1}\right)$ e da concentração total (mmol L $\left.{ }^{-1}\right)$ de alguns ânions e cátions utilizados para estimativa das espécies químicas de alumínio, cálcio, magnésio, fósforo e enxofre e na solução de um Latossolo sob sistema plantio direto em função das doses de gesso e das profundidades de amostragem. Os valores entre parênteses representam o erro padrão da média $(\mathrm{n}=3)$.

\begin{tabular}{|c|c|c|c|c|c|}
\hline \multirow{2}{*}{ Profundidade (m) } & \multirow{2}{*}{ Parâmetros } & \multicolumn{4}{|c|}{ Doses de gesso $\left(\mathrm{t} \mathrm{ha}^{-1}\right)$} \\
\hline & & 0 & 3 & 6 & 9 \\
\hline \multirow[t]{10}{*}{$0-0,05$} & $\mathrm{pH}$ & $4,97 \pm(0,03)$ & $4,83 \pm(0,02)$ & $4,43 \pm(0,11)$ & $5,13 \pm(0,15)$ \\
\hline & I & $4,61 \pm(1,02)$ & $7,12 \pm(2,30)$ & $5,83 \pm(1,14)$ & $4,91 \pm(0,58)$ \\
\hline & COD & $16,76 \pm(0,40)$ & $20,51 \pm(2,15)$ & $19,74 \pm(1,83)$ & $17,41 \pm(1,12)$ \\
\hline & $\mathrm{N}-\mathrm{NO}_{3}^{-}$ & $1,168 \pm(0,040)$ & $1,547 \pm(0,244)$ & $1,425 \pm(0,04)$ & $1,046 \pm(0,206)$ \\
\hline & $\mathrm{Cl}$ & $0,162 \pm(0,040)$ & $0,168 \pm(0,029)$ & $0,173 \pm(0,034)$ & $0,197 \pm(0,073)$ \\
\hline & $\mathrm{F}$ & $0,114 \pm(0,005)$ & $0,129 \pm(0,007)$ & $0,126 \pm(0,006)$ & $0,117 \pm(0,047)$ \\
\hline & $\mathrm{K}$ & $0,816 \pm(0,131)$ & $0,682 \pm(0,062)$ & $0,735 \pm(0,075)$ & $0,687 \pm(0,069)$ \\
\hline & $\mathrm{Na}$ & $0,063 \pm(0,013)$ & $0,07 \pm(0,028)$ & $0,063 \pm(0,023)$ & $0,121 \pm(0,024)$ \\
\hline & $\mathrm{Fe}$ & $0,184 \pm(0,162)$ & $0,104 \pm(0,057)$ & $0,062 \pm(0,027)$ & $0,037 \pm(0,027)$ \\
\hline & $\mathrm{Mn}$ & $0,0032 \pm(0,0003)$ & $0,0035 \pm(0,0004)$ & $0,0038 \pm(0,0004)$ & $0,0025 \pm(0,0009)$ \\
\hline \multirow[t]{10}{*}{$0,05-0,1$} & $\mathrm{pH}$ & $4,80 \pm(0,08)$ & $4,69 \pm(0,09)$ & $4,75 \pm(0,08)$ & $4,85 \pm(0,06)$ \\
\hline & I & $3,39 \pm(0,70)$ & $2,65 \pm(0,43)$ & $3,78 \pm(0,70)$ & $3,27 \pm(0,30)$ \\
\hline & COD & $16,50 \pm(2,52)$ & $14,91 \pm(1,25)$ & $14,98 \pm(1,25)$ & $13,10 \pm(0,74)$ \\
\hline & $\mathrm{N}-\mathrm{NO}_{3}{ }^{-}$ & $0,593 \pm(0,061)$ & $0,521 \pm(0,079)$ & $0,679 \pm(0,084)$ & $0,697 \pm(0,109)$ \\
\hline & $\mathrm{Cl}$ & $0,177 \pm(0,039)$ & $0,116 \pm(0,042)$ & $0,294 \pm(0,107)$ & $0,147 \pm(0,027)$ \\
\hline & $\mathrm{F}$ & $0,076 \pm(0,023)$ & $0,047 \pm(0,026)$ & $0,049 \pm(0,016)$ & $0,037 \pm(0,004)$ \\
\hline & $\mathrm{K}$ & $0,435 \pm(0,060)$ & $0,340 \pm(0,018)$ & $0,354 \pm(0,021)$ & $0,394 \pm(0,068)$ \\
\hline & $\mathrm{Na}$ & $0,056 \pm(0,019)$ & $0,024 \pm(0,004)$ & $0,065 \pm(0,022)$ & $0,044 \pm(0,014)$ \\
\hline & $\mathrm{Fe}$ & $0,032 \pm(0,011)$ & $0,026 \pm(0,010)$ & $0,070 \pm(0,040)$ & $0,073 \pm(0,041)$ \\
\hline & $\mathrm{Mn}$ & $0,0014 \pm(0,0002)$ & $0,0012 \pm(0,0002)$ & $0,0018 \pm(0,0002)$ & $0,0012 \pm(0,0002)$ \\
\hline \multirow[t]{10}{*}{$0,1-0,2$} & $\mathrm{pH}$ & $4,80 \pm(0,08)$ & $4,60 \pm(0,06)$ & $4,62 \pm(0,15)$ & $4,72 \pm(0,15)$ \\
\hline & I & $1,64 \pm(0,20)$ & $2,19 \pm(0,29)$ & $1,66 \pm(0,23)$ & $1,80 \pm(0,10)$ \\
\hline & COD & $12,12 \pm(1,91)$ & $11,96 \pm(0,98)$ & $10,51 \pm(1,26)$ & $8,75 \pm(0,41)$ \\
\hline & $\mathrm{N}-\mathrm{NO}_{3}{ }^{-}$ & $0,584 \pm(0,092)$ & $0,513 \pm(0,106)$ & $0,593 \pm(0,108)$ & $0,626 \pm(0,034)$ \\
\hline & $\mathrm{Cl}$ & $0,149 \pm(0,032)$ & $0,118 \pm(0,032)$ & $0,185 \pm(0,030)$ & $0,142 \pm(0,011)$ \\
\hline & $\mathrm{F}$ & $0,067 \pm(0,009)$ & $0,050 \pm(0,010)$ & $0,066 \pm(0,007)$ & $0,052 \pm(0,013)$ \\
\hline & $\mathrm{K}$ & $0,288 \pm(0,035)$ & $0,238 \pm(0,020)$ & $0,252 \pm(0,008)$ & $0,264 \pm(0,037)$ \\
\hline & $\mathrm{Na}$ & $0,049 \pm(0,020)$ & $0,025 \pm(0,009)$ & $0,040 \pm(0,016)$ & $0,048 \pm(0,015)$ \\
\hline & $\mathrm{Fe}$ & $0,010 \pm(0,006)$ & $0,027 \pm(0,010)$ & $0,011 \pm(0,008)$ & $0,016 \pm(0,005)$ \\
\hline & $\mathrm{Mn}$ & $0,0006 \pm(0,0001)$ & $0,0010 \pm(0,0003)$ & $0,0014 \pm(0,0001)$ & $0,0008 \pm(0,0004)$ \\
\hline \multirow[t]{10}{*}{$0,2-0,4$} & $\mathrm{pH}$ & $4,86 \pm(0,13)$ & $4,66 \pm(0,04)$ & $4,64 \pm(0,15)$ & $4,73 \pm(0,01)$ \\
\hline & I & $1,43 \pm(0,31)$ & $1,24 \pm(0,11)$ & $1,37 \pm(0,04)$ & $1,49 \pm(0,140)$ \\
\hline & COD & $6,44 \pm(1,04)$ & $7,03 \pm(0,51)$ & $7,08 \pm(1,01)$ & $6,46 \pm(1,12)$ \\
\hline & $\mathrm{N}-\mathrm{NO}_{3}{ }^{-}$ & $0,603 \pm(0,026)$ & $0,381 \pm(0,043)$ & $0,535 \pm(0,024)$ & $0,529 \pm(0,037)$ \\
\hline & $\mathrm{Cl}$ & $0,267 \pm(0,104)$ & $0,108 \pm(0,035)$ & $0,107 \pm(0,018)$ & $0,124 \pm(0,023)$ \\
\hline & $\mathrm{F}$ & $0,043 \pm(0,007)$ & $0,044 \pm(0,006)$ & $0,045 \pm(0,004)$ & $0,048 \pm(0,008)$ \\
\hline & $\mathrm{K}$ & $0,220 \pm(0,065)$ & $0,207 \pm(0,021)$ & $0,193 \pm(0,021)$ & $0,197 \pm(0,036)$ \\
\hline & $\mathrm{Na}$ & $0,056 \pm(0,035)$ & $0,037 \pm(0,008)$ & $0,028 \pm(0,011)$ & $0,045 \pm(0,023)$ \\
\hline & $\mathrm{Fe}$ & $0,007 \pm(0,007)$ & $0,007 \pm(0,006)$ & $0,002 \pm(0,001)$ & $0,001 \pm(0,00)$ \\
\hline & $\mathrm{Mn}$ & $0,0011 \pm(0,0003)$ & $0,0077 \pm(0,0060)$ & $0,0022 \pm(0,0002)$ & $0,0018 \pm(0,0002)$ \\
\hline
\end{tabular}

F predominou sobre o par iônico $\mathrm{AlSO}_{4}{ }^{+}$, como constatado por CAMERON et al. (1986). Segundo esses autores, essa diferença acarreta maior efeito do $\mathrm{F}^{-}$em relação ao $\mathrm{S}_{-} \mathrm{SO}_{4}^{-2}$ na redução da toxicidade do $\mathrm{Al}$ para as raízes das plantas.
Os pares iônicos ou os complexos predominaram sobre a forma livre de $\mathrm{Al}$ em todas as doses de gesso e profundidades de amostragem. Mesmo em condições de elevada acidez na solução do solo, que seria uma situação favorável para o Al 
Tabela 2 - Teor total de $\mathrm{Al}\left(\mathrm{Al}_{\mathrm{t}}\right)$ e suas respectivas espécies químicas na solução de um latossolo sob sistema plantio direto em função das doses de gesso e profundidades de amostragem. Os valores entre parênteses representam o erro padrão da média (n = 3).

\begin{tabular}{|c|c|c|c|c|c|c|c|}
\hline \multirow{2}{*}{$\begin{array}{l}\text { Profundidade } \\
\text { (m) }\end{array}$} & \multirow{2}{*}{$\begin{array}{l}\text { Gesso } \\
\left(\mathrm{t} \mathrm{ha}^{-1}\right)\end{array}$} & \multirow{2}{*}{$\frac{\mathrm{Al}_{\mathrm{t}}}{\left(\mathrm{mmol} \mathrm{L}^{-1}\right)}$} & \multicolumn{5}{|c|}{ Espécies de Al (\%) } \\
\hline & & & $\mathrm{Al}^{+3}$ & $\mathrm{Al}-\mathrm{OH}^{(1)}$ & $\mathrm{AlSO}_{4}^{+}$ & $\mathrm{Al}-\mathrm{F}^{(2)}$ & Al-COD \\
\hline \multirow[t]{4}{*}{$0-0,05$} & 0 & $0,25 \pm(0,01)$ & $0,9 \pm(0,3)$ & $0,8 \pm(0,3)$ & $0,1 \pm(0,0)$ & $29,4 \pm(1,1)$ & $68,7 \pm(1,2)$ \\
\hline & 3 & $0,84 \pm(0,38)$ & $7,9 \pm(5,9)$ & $4,2 \pm(3,0)$ & $0,8 \pm(0,5)$ & $19,0 \pm(7,7)$ & $68,2 \pm(6,0)$ \\
\hline & 6 & $0,59 \pm(0,20)$ & $3,3 \pm(2,1)$ & $2,0 \pm(1,4)$ & $0,6 \pm(0,3)$ & $19,5 \pm(4,3)$ & $74,5 \pm(0,6)$ \\
\hline & 9 & $0,39 \pm(0,13)$ & $1,2 \pm(0,3)$ & $2,0 \pm(0,7)$ & $0,2 \pm(0,1)$ & $20,5 \pm(4,7)$ & $76,1 \pm(4,3)$ \\
\hline \multirow[t]{4}{*}{$0,05-0,1$} & 0 & $0,40 \pm(0,14)$ & $1,2 \pm(0,6)$ & $0,9 \pm(0,6)$ & $0,1 \pm(0,0)$ & $17,3 \pm(7,6)$ & $80,4 \pm(6,9)$ \\
\hline & 3 & $0,28 \pm(0,08)$ & $0,9 \pm(0,3)$ & $0,4 \pm(0,2)$ & $0,2 \pm(0,1)$ & $15,9 \pm(9,9)$ & $82,6 \pm(10,2)$ \\
\hline & 6 & $0,43 \pm(0,11)$ & $1,9 \pm(1,0)$ & $0,9 \pm(0,4)$ & $0,3 \pm(0,1)$ & $10,9 \pm(5,1)$ & $86,0 \pm(4,0)$ \\
\hline & 9 & $0,31 \pm(0,04)$ & $1,0 \pm(0,2)$ & $0,7 \pm(0,1)$ & $0,2 \pm(0,0)$ & $9,7 \pm(1,7)$ & $88,4 \pm(1,6)$ \\
\hline \multirow[t]{4}{*}{$0,1-0,2$} & 0 & $0,10 \pm(0,05)$ & $0,2 \pm(0,1)$ & $0,2 \pm(0,1)$ & - & $51,1 \pm(13,9)$ & $48,5 \pm(13,8)$ \\
\hline & 3 & $0,20 \pm(0,08)$ & $0,4 \pm(0,1)$ & $0,1 \pm(0,0)$ & $0,1 \pm(0,0)$ & $39,5 \pm(10,2)$ & $59,9 \pm(10,1)$ \\
\hline & 6 & $0,07 \pm(0,05)$ & $0,2 \pm(0,2)$ & $0,1 \pm(0,1)$ & - & $68,9 \pm(24,7)$ & $30,8 \pm(24,4)$ \\
\hline & 9 & $0,08 \pm(0,04)$ & $0,2 \pm(0,1)$ & $0,2 \pm(0,1)$ & $0,1 \pm(0,0)$ & $50,2 \pm(25,1)$ & $49,2 \pm(25,0)$ \\
\hline \multirow[t]{4}{*}{$0,2-0,4$} & 0 & $0,05 \pm(0,05)$ & $0,1 \pm(0,1)$ & $0,2 \pm(0,2)$ & & $66,1 \pm(25,0)$ & $33,5 \pm(24,6)$ \\
\hline & 3 & $0,02 \pm(0,01)$ & $0,1 \pm(0,1)$ & $0,1 \pm(0,0)$ & $0,1 \pm(0,0)$ & $69,3 \pm(12,9)$ & $30,4 \pm(12,8)$ \\
\hline & 6 & $0,01 \pm(0,01)$ & $0,1 \pm(0,0)$ & - & - & $85,8 \pm(8,7)$ & $14,1 \pm(8,7)$ \\
\hline & 9 & $0,01 \pm(0,01)$ & $0,1 \pm(0,1)$ & - & - & $85,5 \pm(11,1)$ & $14,4 \pm(11,0)$ \\
\hline
\end{tabular}

${ }^{(1)} \mathrm{Al}-\mathrm{OH}=\mathrm{Al}(\mathrm{OH})^{+2}+\mathrm{Al}(\mathrm{OH})^{+}+\mathrm{Al}(\mathrm{OH})_{3}^{0}{ }^{(2)} \mathrm{Al}-\mathrm{F}=\mathrm{Al}(\mathrm{F})^{+2}+\mathrm{Al}(\mathrm{F})^{+}+\mathrm{Al}(\mathrm{F})^{0}{ }_{3}$

encontrar-se na forma mais tóxica às plantas $\left(\mathrm{Al}^{+3}\right)(\mathrm{MA}$ \& FURUKAWA, 2003), essa espécie química ocorreu em baixas porcentagens. A máxima proporção ocupada pela forma livre foi 7,9\% na camada de $0-0,05 \mathrm{~m}$, no tratamento com aplicação de $3 t h^{-1}$ de gesso (Tabela 2).

O COD foi o principal ligante para o Ca e o $\mathrm{Mg}$, mas em muito maior magnitude para o primeiro (Tabela 4). Esta constatação confirma a já conhecida importância de complexos orgânicos hidrossolúveis na dinâmica desses nutrientes em solos com acúmulo de MO, como observado para o Ca por FRANCHINI et al. (1999). Para esses autores, o Ca, na sua forma complexada, teria sua carga líquida alterada pela formação de complexos de carga nula ou até negativa, o que favoreceria a sua lixiviação. Apesar de o par iônico $\mathrm{CaSO}_{4}{ }^{0}$ não representar mais que $1 \%$ de todo o $\mathrm{Ca}$ contido na solução do solo, o incremento de $\mathrm{S}_{-} \mathrm{SO}_{4}^{-2}$ aumentou a presença de $\mathrm{CaSO}_{4}{ }^{0}$, o que foi confirmado pelas correlações entre eles $(r=099, P=0,01 ; r=0,93$, $\mathrm{P}=0,03 ; \mathrm{r}=0,99, \mathrm{P}=0,01$ para as profundidades de 0 0,05; 0,05-0,1 e 0,2-0,4m, respectivamente). A especiação do Ca foi regulada, principalmente, pela disponibilidade de COD para a formação de complexos. Até $0,1 \mathrm{~m}$, a forma livre $\mathrm{Ca}^{+2}$ predominou sobre o Ca-COD (Tabela
4). Essa diferença ocorreu devido à maior competição com outros cátions para a ligação com COD, e dos maiores teores totais de Ca solúvel presentes até esta profundidade, o que favoreceu a presença da forma livre $\mathrm{Ca}^{+2}$.

Para o Mg, a ocorrência da forma livre $\mathrm{Mg}^{+2}$ foi muito maior do que a sua associação com ânions orgânicos e inorgânicos (Tabela 4). O ânion inorgânico que mais se, ligou ao $\mathrm{Mg}$ foi o $\mathrm{S}_{-} \mathrm{SO}_{4}^{-2}$, o que ajuda a explicar por que a aplicação de gesso pode favorecer a lixiviação de Mg (QUAGGIO et al., 1982; ROSOLEM \& MACHADO, 1984), uma vez, que para ocorrer a movimentação de bases em solos, é necessária a presença de ânions acompanhantes (RAIJ, 1988). A interação destes dois elementos foi caracterizada pelas correlações entre os teores de S-SO ${ }_{4}^{-2}$ e do par iônico $\mathrm{MgSO}_{4}{ }^{0}(\mathrm{r}=0,99, \mathrm{P}=0,01 ; \mathrm{r}=0,99, \mathrm{P}=0,005 ; \mathrm{r}=1,0, \mathrm{P}$ $=0,004$ para as profundidades de 0,05-0,1; 0,1-0,2 e 0,2$0,4 \mathrm{~m}$, respectivamente).

Além de os teores de P na solução do solo terem sido muito baixos, houve intensa associação desse nutriente com cátions (Tabela 3), principalmente na primeira camada, em que somente no tratamento com ausência de gesso a proporção ocupada pela forma livre $\mathrm{H}_{2} \mathrm{PO}_{4}^{-}$foi maior do que o fósforo solúvel

Ciência Rural, v.37, n.1, jan-fev, 2007. 
Tabela 3 - Teores totais de fosfato $\left(\mathrm{P}_{\mathrm{t}}-\mathrm{H}_{\mathrm{x}} \mathrm{PO}_{4}{ }^{\mathrm{x}}\right)$, de sulfato $\left(\mathrm{S}_{\mathrm{t}}-\mathrm{SO}_{4}{ }^{-2}\right)$ e suas respectivas espécies químicas na solução de um latossolo sob sistema plantio direto em função das doses de gesso e das profundidades de amostragem. Os valores entre parênteses representam o erro padrão da média $(n=3)$.

\begin{tabular}{|c|c|c|c|c|c|c|}
\hline \multirow[t]{2}{*}{$\begin{array}{l}\text { Profundidade } \\
\text { (m) }\end{array}$} & \multirow[t]{2}{*}{$\begin{array}{l}\text { Gesso } \\
\left(\mathrm{t} \mathrm{ha}^{-1}\right)\end{array}$} & \multirow{2}{*}{$\begin{array}{c}\left.\begin{array}{c}\text { Teor total } \\
(\mathrm{mmol} \mathrm{L}\end{array}\right) \\
\mathrm{P}_{\mathrm{t}}-\mathrm{H}_{\mathrm{x}} \mathrm{PO}_{4}{ }^{\mathrm{x}}\end{array}$} & \multicolumn{4}{|c|}{$\begin{array}{c}\text { Espécies } \\
(\%)\end{array}$} \\
\hline & & & $\mathrm{HPO}_{4}^{-2}$ & $\mathrm{H}_{2} \mathrm{PO}_{4}^{-}$ & $\mathrm{Al}-\mathrm{H}_{\mathrm{x}} \mathrm{PO}_{4}{ }^{\mathrm{x}}$ & Outras $^{(1)}$ \\
\hline \multirow[t]{4}{*}{$0-0,05$} & 0 & $0,0011 \pm(0,0016)$ & $0,5 \pm(0,1)$ & $71,4 \pm(19,3)$ & $18,6 \pm(5,7)$ & $9,5 \pm(3,7)$ \\
\hline & 3 & $0,0001 \pm(0,0001)$ & $0,2 \pm(0,2)$ & $38,2 \pm(44,1)$ & $59,8 \pm(34,0)$ & $1,9 \pm(1,2)$ \\
\hline & 6 & $0,0001 \pm(0,0001)$ & $0,3 \pm(0,2)$ & $50,3 \pm(36,0)$ & $47,2 \pm(36,2)$ & $2,2 \pm(0,4)$ \\
\hline & 9 & $0,0001 \pm(0,0001)$ & $0,7 \pm(0,5)$ & $54,2 \pm(17,1)$ & $42,6 \pm(19,7)$ & $2,6 \pm(2,9)$ \\
\hline \multirow[t]{4}{*}{$0,05-0,1$} & 0 & $0,0140 \pm(0,0009)$ & $0,3 \pm(0,0)$ & $67,2 \pm(19,0)$ & $31,0 \pm(18,8)$ & $1,5 \pm(0,3)$ \\
\hline & 3 & $0,0002 \pm(0,0001)$ & $0,3 \pm(0,0)$ & $79,9 \pm(9,2)$ & $18,3 \pm(8,8)$ & $1,5 \pm(0,4)$ \\
\hline & 6 & nd* & - & - & - & - \\
\hline & 9 & nd & - & - & - & - \\
\hline \multirow[t]{4}{*}{$0,1-0,2$} & 0 & $0,0001 \pm(0,0000)$ & $0,5 \pm(0,1)$ & $95,7 \pm(2,7)$ & $3,2 \pm(2,6)$ & $0,7 \pm(0,2)$ \\
\hline & 3 & nd & - & - & - & - \\
\hline & 6 & $0,0006 \pm(0,0004)$ & $0,3 \pm(0,1)$ & $96,2 \pm(2,7)$ & $2,6 \pm(2,5)$ & $0,9 \pm(0,2)$ \\
\hline & 9 & $0,0002 \pm(0,0002)$ & $0,4 \pm(0,1)$ & $94,9 \pm(2,7)$ & $3,6 \pm(2,6)$ & $1,0 \pm(0,2)$ \\
\hline \multirow[t]{5}{*}{$0,2-0,4$} & 0 & $0,0002 \pm(0,0002)$ & $0,5 \pm(0,2)$ & $96,0 \pm(3,1)$ & $2,8 \pm(2,8)$ & $0,6 \pm(0,2)$ \\
\hline & 3 & $0,0009 \pm(0,0009)$ & $0,3 \pm(0,0)$ & $98,7 \pm(0,4)$ & $0,4 \pm(0,2)$ & $0,7 \pm(0,2)$ \\
\hline & 6 & $0,0003 \pm(0,0002)$ & $0,3 \pm(0,1)$ & $98,9 \pm(0,1)$ & $0,0 \pm(0,0)$ & $0,7 \pm(0,1)$ \\
\hline & 9 & nd & - & - & - & - \\
\hline & & $\mathrm{S}_{\mathrm{t}}-\mathrm{SO}_{4}^{-2}$ & $\mathrm{SO}_{4}^{-2}$ & $\mathrm{MgSO}_{4}{ }^{0}$ & $\mathrm{CaSO}_{4}{ }^{0}$ & Outras $^{(2)}$ \\
\hline \multirow[t]{4}{*}{$0-0,05$} & 0 & $0,057 \pm(0,004)$ & $93,5 \pm(0,4)$ & $3,1 \pm(0,1)$ & $2,3 \pm(0,1)$ & $1,1 \pm(0,2)$ \\
\hline & 3 & $0,072 \pm(0,003)$ & $91,3 \pm(1,9)$ & $2,0 \pm(0,2)$ & $3,0 \pm(0,3)$ & $3,7 \pm(1,6)$ \\
\hline & 6 & $0,089 \pm(0,009)$ & $88,6 \pm(4,2)$ & $2,0 \pm(0,1)$ & $3,8 \pm(0,3)$ & $5,7 \pm(3,9)$ \\
\hline & 9 & $0,070 \pm(0,018)$ & $91,3 \pm(0,8)$ & $2,5 \pm(0,3)$ & $4,4 \pm(0,3)$ & $1,8 \pm(0,7)$ \\
\hline \multirow[t]{4}{*}{$0,05-0,1$} & 0 & $0,026 \pm(0,003)$ & $94,6 \pm(1,3)$ & $1,8 \pm(0,1)$ & $1,5 \pm(0,1)$ & $2,2 \pm(1,2)$ \\
\hline & 3 & $0,034 \pm(0,005)$ & $95,7 \pm(0,5)$ & $1,2 \pm(0,1)$ & $1,8 \pm(0,1)$ & $1,3 \pm(0,4)$ \\
\hline & 6 & $0,053 \pm(0,003)$ & $93,9 \pm(1,9)$ & $0,8 \pm(0,0)$ & $2,3 \pm(0,2)$ & $3,0 \pm(1,7)$ \\
\hline & 9 & $0,062 \pm(0,004)$ & $95,4 \pm(0,3)$ & $0,7 \pm(0,1)$ & $2,5 \pm(0,1)$ & $1,3 \pm(0,3)$ \\
\hline \multirow[t]{4}{*}{$0,1-0,2$} & 0 & $0,015 \pm(0,003)$ & $97,1 \pm(0,2)$ & $1,7 \pm(0,1)$ & $0,8 \pm(0,1)$ & $0,4 \pm(0,1)$ \\
\hline & 3 & $0,030 \pm(0,005)$ & $96,7 \pm(0,3)$ & $1,3 \pm(0,1)$ & $1,5 \pm(0,1)$ & $0,6 \pm(0,1)$ \\
\hline & 6 & $0,065 \pm(0,008)$ & $97,1 \pm(0,2)$ & $0,7 \pm(0,0)$ & $1,7 \pm(0,1)$ & $0,5 \pm(0,1)$ \\
\hline & 9 & $0,066 \pm(0,009)$ & $97,0 \pm(0,4)$ & $0,6 \pm(0,2)$ & $1,9 \pm(0,3)$ & $0,5 \pm(0,1)$ \\
\hline \multirow[t]{4}{*}{$0,2-0,4$} & 0 & $0,010 \pm(0,002)$ & $97,4 \pm(0,1)$ & $1,6 \pm(0,1)$ & $0,7 \pm(0,1)$ & $0,3 \pm(0,0)$ \\
\hline & 3 & $0,073 \pm(0,006)$ & $97,2 \pm(0,4)$ & $1,2 \pm(0,1)$ & $1,1 \pm(0,2)$ & $0,4 \pm(0,1)$ \\
\hline & 6 & $0,095 \pm(0,016)$ & $97,0 \pm(0,2)$ & $1,0 \pm(0,1)$ & $1,6 \pm(0,2)$ & $0,4 \pm(0,1)$ \\
\hline & 9 & $0,123 \pm(0,022)$ & $97,1 \pm(0,3)$ & $0,5 \pm(0,3)$ & $1,4 \pm(0,5)$ & $1,1 \pm(0,8)$ \\
\hline
\end{tabular}

${ }^{(1)}$ Outras espécies de fosfato: $\mathrm{Ca}-\mathrm{H}_{\mathrm{x}} \mathrm{PO}_{4}{ }^{\mathrm{x}}, \mathrm{Mg}-\mathrm{H}_{\mathrm{x}} \mathrm{PO}_{4}{ }^{\mathrm{x}}, \mathrm{Mn}-\mathrm{H}_{\mathrm{x}} \mathrm{PO}_{4}{ }^{\mathrm{x}}, \mathrm{Fe}-\mathrm{H}_{\mathrm{x}} \mathrm{PO}_{4}{ }^{\mathrm{x}} ; \mathrm{KH}_{2} \mathrm{PO}_{4}$. ${ }^{(2)}$ Outras espécies de sulfato: $\mathrm{AlSO}_{4}{ }^{+}, \mathrm{KSO}_{4}{ }^{-}$, $\mathrm{MnSO}_{4}{ }^{0}, \mathrm{NaSO}_{4}$.

*nd: concentração de fosfato na solução do solo não detectada.

combinado com o Al (Al- $\left.\mathrm{H}_{\mathrm{x}} \mathrm{PO}_{4}^{\mathrm{x}}\right)$. Com o aumento dos teores de Ca no solo após a gessagem, ocorreu maior deslocamento de Al do complexo de troca para a solução, incrementando a proporção ocupada pela espécie $\mathrm{AlH}_{\mathrm{x}} \mathrm{PO}_{4}{ }^{\mathrm{x}}$. Entretanto, logo na segunda camada, mesmo com a gessagem, a forma livre $\mathrm{H}_{2} \mathrm{PO}_{4}^{-}$foi predominante, o que se estendeu para as camadas subjacentes. Essas espécies são importantes de serem conhecidas porque a atividade ou a concentração de íons fosfatos na solução do solo e, portanto, a 
Tabela 4 - Teores totais de cálcio $\left(\mathrm{Ca}_{\mathrm{t}}\right)$, de magnésio $\left(\mathrm{Mg}_{\mathrm{t}}\right)$ e suas respectivas espécies químicas na solução de um latossolo sob sistema plantio direto em função das doses de gesso e das profundidades de amostragem. Os valores entre parênteses representam o erro padrão da média $(n=3)$.

\begin{tabular}{|c|c|c|c|c|c|c|}
\hline \multirow{2}{*}{ Profundidade (m) } & \multirow{2}{*}{$\frac{\text { Gesso }}{\left(\mathrm{t} \mathrm{ha}^{-1}\right)}$} & \multirow{2}{*}{$\frac{\text { Teor total }}{\left(\mathrm{mmol} \mathrm{L}^{-1}\right)}$} & \multicolumn{4}{|c|}{ Espécies } \\
\hline & & & & & & \\
\hline & & $\mathrm{Ca}_{\mathrm{t}}$ & $\mathrm{Ca}^{+2}$ & Ca-COD & $\mathrm{CaSO}_{4}{ }^{0}$ & Outras $^{(1)}$ \\
\hline \multirow[t]{4}{*}{$0-0,05$} & 0 & $0,32 \pm(0,02)$ & $58,8 \pm(7,0)$ & $40,6 \pm(7,1)$ & $0,4 \pm(0,1)$ & $0,2 \pm(0,1)$ \\
\hline & 3 & $0,43 \pm(0,04)$ & $77,7 \pm(12,9)$ & $21,5 \pm(13,0)$ & $0,5 \pm(0,1)$ & $0,3 \pm(0,1)$ \\
\hline & 6 & $0,48 \pm(0,04)$ & $74,6 \pm(8,8)$ & $24,4 \pm(8,9)$ & $0,7 \pm(0,1)$ & $0,3 \pm(0,1)$ \\
\hline & 9 & $0,60 \pm(0,01)$ & $64,7 \pm(6,1)$ & $34,6 \pm(6,1)$ & $0,5 \pm(0,1)$ & $0,2 \pm(0,1)$ \\
\hline \multirow[t]{4}{*}{$0,05-0,1$} & 0 & $0,19 \pm(0,01)$ & $61,6 \pm(8,5)$ & $38,1 \pm(8,5)$ & $0,2 \pm(0,0)$ & $0,1 \pm(0,0)$ \\
\hline & 3 & $0,23 \pm(0,01)$ & $58,3 \pm(4,6)$ & $41,3 \pm(4,6)$ & $0,3 \pm(0,0)$ & $0,1 \pm(0,1)$ \\
\hline & 6 & $0,30 \pm(0,01)$ & $67,8 \pm(8,2)$ & $31,6 \pm(8,2)$ & $0,5 \pm(0,0)$ & $0,2 \pm(0,1)$ \\
\hline & 9 & $0,32 \pm(0,00)$ & $60,6 \pm(4,1)$ & $38,8 \pm(4,2)$ & $0,5 \pm(0,1)$ & $0,1 \pm(0,1)$ \\
\hline \multirow[t]{4}{*}{$0,1-0,2$} & 0 & $0,14 \pm(0,02)$ & $39,7 \pm(2,3)$ & $60,2 \pm(2,3)$ & $0,1 \pm(0,0)$ & $0,1 \pm(0,0)$ \\
\hline & 3 & $0,20 \pm(0,01)$ & $50,0 \pm(3,0)$ & $49,7 \pm(2,9)$ & $0,3 \pm(0,0)$ & $0,1 \pm(0,0)$ \\
\hline & 6 & $0,23 \pm(0,01)$ & $47,3 \pm(5,0)$ & $52,1 \pm(5,1)$ & $0,5 \pm(0,1)$ & $0,1 \pm(0,1)$ \\
\hline & 9 & $0,28 \pm(0,03)$ & $45,1 \pm(2,9)$ & $54,3 \pm(3,0)$ & $0,5 \pm(0,1)$ & $0,1 \pm(0,1)$ \\
\hline \multirow[t]{5}{*}{$0,2-0,4$} & 0 & $0,12 \pm(0,00)$ & $36,2 \pm(1,7)$ & $63,7 \pm(1,7)$ & $0,1 \pm(0,0)$ & $0,1 \pm(0,0)$ \\
\hline & 3 & $0,17 \pm(0,02)$ & $41,4 \pm(2,2)$ & $58,1 \pm(2,3)$ & $0,5 \pm(0,0)$ & $0,1 \pm(0,1)$ \\
\hline & 6 & $0,23 \pm(0,02)$ & $43,6 \pm(4,9)$ & $55,6 \pm(5,1)$ & $0,7 \pm(0,2)$ & $0,1 \pm(0,0)$ \\
\hline & 9 & $0,30 \pm(0,02)$ & $41,9 \pm(1,2)$ & $57,3 \pm(1,4)$ & $0,8 \pm(0,1)$ & $0,1 \pm(0,0)$ \\
\hline & & $\mathrm{Mg}_{\mathrm{t}}$ & $\mathrm{Mg}^{+2}$ & Mg-COD & $\mathrm{MgSO}_{4}{ }^{0}$ & Outras $^{(2)}$ \\
\hline \multirow[t]{4}{*}{$0-0,05$} & 0 & $0,36 \pm(0,03)$ & $92,7 \pm(1,6)$ & $6,7 \pm(1,6)$ & $0,5 \pm(0,0)$ & $0,1 \pm(0,0)$ \\
\hline & 3 & $0,30 \pm(0,04)$ & $96,1 \pm(2,4)$ & $3,4 \pm(2,4)$ & $0,5 \pm(0,1)$ & $0,1 \pm(0,0)$ \\
\hline & 6 & $0,24 \pm(0,02)$ & $95,8 \pm(1,5)$ & $3,4 \pm(1,5)$ & $0,7 \pm(0,1)$ & $0,1 \pm(0,0)$ \\
\hline & 9 & $0,30 \pm(0,02)$ & $94,1 \pm(1,3)$ & $5,3 \pm(1,3)$ & $0,6 \pm(0,2)$ & $0,1 \pm(0,0)$ \\
\hline \multirow[t]{4}{*}{$0,05-0,1$} & 0 & $0,18 \pm(0,01)$ & $93,4 \pm(1,9)$ & $6,2 \pm(1,8)$ & $0,3 \pm(0,0)$ & $0,1 \pm(0,0)$ \\
\hline & 3 & $0,11 \pm(0,01)$ & $92,9 \pm(1,1)$ & $6,7 \pm(1,1)$ & $0,4 \pm(0,0)$ & $0,1 \pm(0,0)$ \\
\hline & 6 & $0,09 \pm(0,01)$ & $94,7 \pm(1,5)$ & $4,7 \pm(1,5)$ & $0,5 \pm(0,0)$ & $0,1 \pm(0,0)$ \\
\hline & 9 & $0,07 \pm(0,01)$ & $93,2 \pm(1,0)$ & $6,1 \pm(1,1)$ & $0,6 \pm(0,0)$ & $0,0 \pm(0,0)$ \\
\hline \multirow[t]{4}{*}{$0,1-0,2$} & 0 & $0,16 \pm(0,01)$ & $86,5 \pm(1,1)$ & $13,2 \pm(1,2)$ & $0,2 \pm(0,0)$ & $0,1 \pm(0,0)$ \\
\hline & 3 & $0,12 \pm(0,01)$ & $90,5 \pm(1,0)$ & $9,1 \pm(1,0)$ & $0,4 \pm(0,0)$ & $0,1 \pm(0,0)$ \\
\hline & 6 & $0,07 \pm(0,01)$ & $88,9 \pm(2,0)$ & $10,2 \pm(2,0)$ & $0,7 \pm(0,1)$ & $0,2 \pm(0,1)$ \\
\hline & 9 & $0,06 \pm(0,01)$ & $88,4 \pm(1,0)$ & $10,8 \pm(1,1)$ & $0,7 \pm(0,1)$ & $0,2 \pm(0,1)$ \\
\hline \multirow[t]{4}{*}{$0,2-0,4$} & 0 & $0,15 \pm(0,01)$ & $84,7 \pm(1,0)$ & $15,0 \pm(0,9)$ & $0,1 \pm(0,0)$ & $0,2 \pm(0,0)$ \\
\hline & 3 & $0,11 \pm(0,01)$ & $86,8 \pm(1,1)$ & $12,3 \pm(1,0)$ & $0,8 \pm(0,1)$ & $0,2 \pm(0,1)$ \\
\hline & 6 & $0,09 \pm(0,01)$ & $87,2 \pm(1,8)$ & $11,5 \pm(1,9)$ & $1,1 \pm(0,2)$ & $0,2 \pm(0,0)$ \\
\hline & 9 & $0,06 \pm(0,02)$ & $86,6 \pm(0,4)$ & $11,9 \pm(0,6)$ & $1,3 \pm(0,2)$ & $0,2 \pm(0,1)$ \\
\hline
\end{tabular}

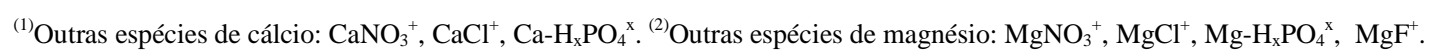

disponibilidade de fósforo aos vegetais, vai depender da proporção das diversas formas de $\mathrm{P}$ e do $\mathrm{pH}$ do sistema (CATANI \& BATAGLIA, 1968).

A forma livre $\mathrm{SO}^{-2}$ foi a espécie química predominante de sulfato ${ }^{4}$ na solução do solo, independentemente do tratamento ou da profundidade de amostragem (Tabela 3). Quando formou par iônico, considerando-se os valores médios das doses de gesso e das profundidades de amostragem, a maior proporção foi ocupada pelo $\mathrm{CaSO}_{4}{ }^{0}$ (2,0\%). A formação de pares

Ciência Rural, v.37, n.1, jan-fev, 2007. 
iônicos pode interferir na disponibilidade de $\mathrm{S}$ às plantas, conforme WONG (1999), que encontrou pequena absorção desse nutriente por plantas de soja, o que foi explicado pela baixa disponibilidade de sulfato na solução do solo e a formação de pares iônicos com $\mathrm{Mn}, \mathrm{Ca}, \mathrm{Mg}$ e Al.

\section{CONCLUSÕES}

A importância do gesso agrícola em amenizar os efeitos fitotóxicos do Al parece ser de menor importância em solos sob SPD, devido à intensa complexação desse cátion pelo COD. O COD apresentou forte interação com Ca e Mg, indicando importante participação na dinâmica desses nutrientes em áreas cultivadas sob SPD. Além de os teores totais de fosfato na solução do solo serem muito baixos, a sua disponibilidade pode ser afetada devido à interação com os cátions presentes na solução do solo, principalmente com o alumínio.

\section{AGRADECIMENTOS}

Ao Conselho Nacional de Desenvolvimento Científico e Tecnológico (CNPq), pela concessão da bolsa de estudo ao primeiro autor e de produtividade em pesquisa aos demais autores. À Fundação de Amparo à Pesquisa do Estado de São Paulo (FAPESP), pelo apoio financeiro para quantificação dos íons em solução.

\section{REFERÊNCIAS}

BARRA, C.M. et al. Especiação de arsênio - uma revisão. Química Nova, v.23, p.58-69, 2000.

CAIRES, E.F. et al. Alterações de características químicas do solo e resposta da soja ao calcário e gesso aplicados na superfície em sistema de cultivo sem preparo do solo. Revista Brasileira de Ciência do Solo, v.22, p.27-34, 1998.

CAMARGO, O.A. et al. Reações de micronutrientes e elementos tóxicos no solo. In: FERREIRA, M.E. et al. Micronutrientes e elementos tóxicos na agricultura. Jaboticabal: Funepe, 2001. Cap.5, p.89-124.

CAMERON, R.S. et al. Relative toxicities of inorganic aluminum complexes to barley. Soil Science Society American Journal, v.50, p.1231-1236, 1986.

CATANI, R.A.; BATAGLIA. O.C. Formas de ocorrência do fósforo no solo latossólico roxo. Anais da Escola Superior da “Luiz de Queiroz”, v.25, p.99-119, 1968.

CHAVES, J.C. et al. Especiação química da solução do solo para interpretação da absorção de cálcio e alumínio por raízes de cafeeiro. Pesquisa Agropecuária Brasileira, v.26, p.447453, 1991.

EMPRESA BRASILEIRA DE PESQUISA AGROPECUÁRIA. Centro Nacional de Pesquisa de Solos. Manual de análises químicas de solos, plantas e fertilizantes. 2.ed. Rio de Janeiro, 1997. 212p.

FRANCHINI, J.C. et al. Dinâmica de íons em solo ácido lixiviado com extratos de resíduos de adubos verdes e soluções puras de ácidos orgânicos. Pesquisa Agropecuária Brasileira, v.34, p.2267-2276, 1999.

GUSTAFSSON, J.P. Visual MINTEQ. Capturado em de 26 de out. 2004. Online. Disponível na Internet: http://www.lwr.kth.se/ English/OurSoftware/ Vminteq .

HENS, M.; MERCKX, R. The role of colloidal particles in the speciation and analysis of "dissolved" phosphorus. Water Research, v.36, p.1483-1492, 2002

MA, J.F.; FURUKAWA, J. Recent progress in the research of external Al detoxification in higher plants: a minireview. Journal of Inorganic Biochemistry, v.97, p.46-51, 2003.

PAVAN, M.A. et al. Manual de análise química do solo e controle de qualidade. Londrina: Instituto Agronômico do Paraná, 1992. 38p. (Circular 76).

QUAGGIO, J.A. et al. Efeitos da aplicação de calcário e gesso sobre a produção de amendoim e a lixiviação de bases no solo. Revista Brasileira de Ciência do Solo, v.6, p.189-194, 1982.

RAIJ, B. van. Gesso agrícola na melhoria do ambiente radicular no subsolo. São Paulo: Anda, 1988. 88p.

RAIJ, B. van. Reações de gesso em solos ácidos. In: SEMINÁRIO SOBRE O USO DO GESSO NA AGRICULTURA, 2., 1992, Uberaba, MG. Anais... Uberaba: Ibrafos, 1992. p.105120 .

RAIJ, B. van. et al. Gesso na produção de cultivares de milho com tolerância diferencial a alumínio em três níveis de calagem. Revista Brasileira de Ciência do Solo, v.22, p.101-108, 1998.

RITCHEY, K.D. et al. Calcium leaching to increase rooting depth in a Brazilian Savannah Oxisol. Agronomy Journal, v.72, p.40-44, 1980.

ROSOLEM, C.A.; MACHADO, J.R. Efeito da calagem e gessagem na produção de algodão e na lixiviação de bases em dois latossolos. Revista Brasileira de Ciência do Solo, v.8, p.103-109, 1984.

SALET, L.R. et al. Atividade do alumínio na solução de solo no sistema de plantio direto. Revista Científica Unicruz, v.1, p.9-13, 1999.

SPOSITO, G. The chemistry of soils. New York: Oxford Univesity, 1989. 277p.

WOLT, J.D. Obtaining soil solution: laboratory methods. In: Soil solution chemistry: applications to environmental science and agriculture. New York: John Wiley, 1994. p.95-120.

WONG, J. Disponibilidade de nutrientes e produção de soja nos solos de Mato Grosso do Sul em função da calagem. 1999. 91f. Dissertação (Mestrado em Agronomia) - Curso de Pós-graduação em Agricultura, Faculdade de Ciências Agronômicas, Universidade Estadual Paulista "Júlio de Mesquita Filho”. 